\title{
Finite element modelling of the dynamics of groovy ball bearings
}

\author{
Olamide Ajala ${ }^{1, \star}$, Ekaterina Pavlovskaia ${ }^{1, \star \star}$, and Marian Weircigroch ${ }^{1, \star \star \star}$ \\ ${ }^{1}$ Centre for Applied Dynamics Research, School of Engineering, University of Aberdeen, UK
}

\begin{abstract}
Geometry modified thrust bearings exposed to rolling and sliding contact are subjected to wear and localized frictional heating caused by relative slip between the two sliding surfaces. This leads to a rise in temperature, thermal stresses and changes in the elastic and plastic strength and physical properties of the material. The changes in the properties in turn alter the stress state, the displacement field, life and reliability of the bearing. Hence, a finite-element model is created to study the dynamics of groovy thrust bearing. In this paper, Hertz contact theory and numerical method are used to simulate the dynamics and kinematics of groovy ball bearings. The optimal loading parameters are identified in this study based on the analysis of the system responses and properties. The results of the numerical analysis and validation are presented. The numerical analysis proves the concept of transforming rotational motion into axial oscillation and demonstrates the capabilities of the numerical simulation to accurately model the dynamics of the groovy ball bearing.
\end{abstract}

\section{Introduction}

Drilling through hard rock has been a restraining factor to economically develop significant hydrocarbon reserves in several geological settings. The high cost to explore and develop these reserves is of considerable importance. Thus, Resonance Enhanced Drilling (RED), was developed at the University of Aberdeen to overcome this challenge [1]. This current research is focused on contact interaction between rolling element bearings (REB), which serve as the main mechanical component of RED module designed to operate under high frequency dynamic rotary action and static preload. This study, aims to develop an ultimate tool for optimal design of the dynamic exciter of RED module [2].

\section{Finite element contact mechanics of groovy bearing}

The exciter is modelled in Abaqus, as a solid continuum body represented by a 3-D finite-element mesh, with encastre and displacement boundary conditions on the cage and race respectively to simulate the exciter operation under dynamic load. First order, 8-node linear brick, hourglass control, reduced integration, 3-D stressdisplacement elements and second order tetrahedral 3-D modified elements (C3D10M) were adopted (see figure 1). A total of 346,557 elements were used to model the assembly in Abaqus. All the parts of the modelled exciter, excluding the balls, are drawn using SolidWorks and subsequently imported into Abaqus, to ensure accurate geometry representation.

\footnotetext{
^e-mail: olamide.sherifah.ajala@abdn.ac.uk

$\star \star$ e-mail: e.pavlovskaia@abdn.ac.uk

$\star \star \star$ e-mail: m.wiercigroch@abdn.ac.uk
}

The balls are constrained in translation in both lateral and longitudinal directions and the top race is constrained in all degrees of freedom (DOF) with exception of translation and rotation in the vertical direction. The Bottom race is constrained in all DOF with the exception of rotation in the vertical direction, while the spring is constrained in all DOF with exception of the translation in vertical direction. The connection between the disc and bearing is modelled using a spring element with spring stiffness of $6.67 \mathrm{kN} / \mathrm{mm}$. The geometry and excitation loads for the selected bearing is presented in table 1 .

The contact condition is simulated by adapting general contact algorithm and finite-sliding contact evolution discretisation to avoid missing contacts. The interactions between the bearing elements are defined with a normal hard pressure overclosure relationship and a penalty constraint enforcement formulation to avoid penetration of the contacting surface.

The model considers both frictionless and isotropic Coulomb friction formulations to simulate the lubricant behaviour of the bearing. Static instabilities in the model, are controlled by adjusting the initial contact states, the external excitation sequence and stiffness damping for numerical accuracy.

\section{Numerical analysis and experimental verification}

The groovy bearing described in this paper adopts a standard thrust ball bearing in which the driven track is modified as $\mathrm{N}$ number of sinusoid over which the rolling elements pass to periodically increase and decrease axial distance between the rotation element and base element while rotating, thereby creating an oscillatory axial motion 
(a)

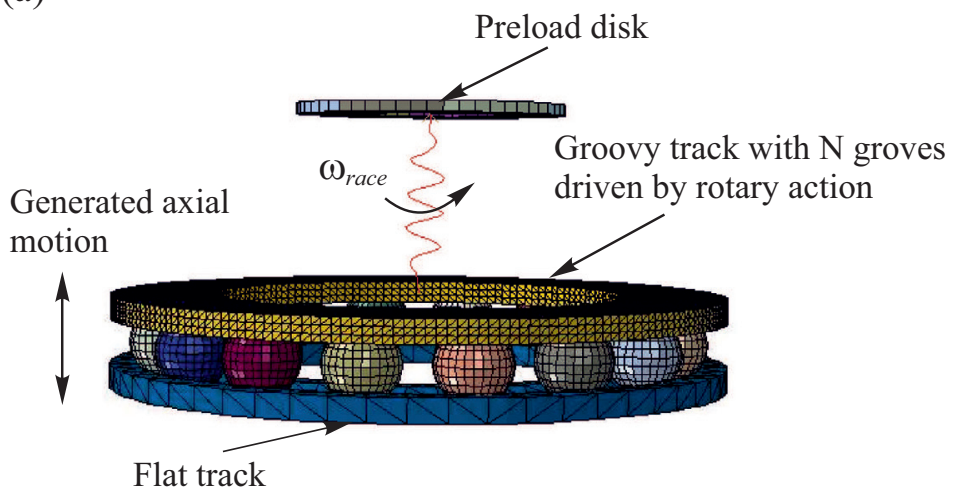

(b)

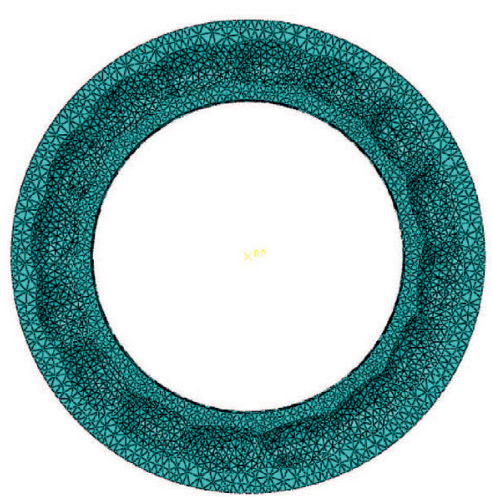

Figure 1. Schematic of the numerical model with C3D10M elements. (a) Main component of the modelled exciter; (b) Close up view of the groovy top race, with $\mathrm{N}$ grooves.

on the rotation element relative to the base element. This arrangement makes the dynamic analysis of the contact interaction between the rolling elements challenging from modelling perspective.

To obtain a bounded solution for adequate analysis of the model characteristics, the stability limit $\left(\Delta t_{\min }\right)$ of the assembly is estimated in terms of the highest eigenvalue $\left(\omega_{\max }\right)$ of the assembly and the fraction of critical damping $(\xi)$ in the highest mode as shown in Eq. (1) [4]:

$$
\Delta t_{\min } \leq \frac{2}{\omega_{\max }}\left(\sqrt{1+\xi^{2}}-\xi\right) .
$$

To evaluate the accuracy of the numerical simulation, the energy balance of the assembly is reviewed for the entire model considering the energy balance equation in Eq. (2). For an accurate finite element analysis, the artificial energy ( $E_{V D}$ and $E_{F D}$ ) of the model should be less than 10 percent of the real energy $\left(E_{K E}\right.$ and $\left.E_{I}\right)$ of the system and the total energy of the system be constant $E_{T}$ ( i.e law of conservation of energy). Problems indicators when reviewing the energy balance of the model are excessive artificial strain energy due to hourglassing of the finite elements and a large change in the total energy $E_{T}$ due to conflicting constraints and exceeding the stability limit of the model:

$$
E_{I}+E_{V D}+E_{F D}+E_{K E}=E_{T} .
$$

\subsection{Model characteristics and analysis}

Dimension and properties of the bearing analysed are listed in table 1 . Using the configuration of the FE model described in previous section (see Sect. 2), series of analysis is performed on the groovy bearing. First, the model is subjected to preload of $6.4 \mathrm{kN}$, that is, downward force is applied to the preload disc shown in figure 1 .

Once the assembly reaches stable value of preload, angular velocity is applied to the top plate of the bearing (one with the sinusoidal profile). As the nominal rotational velocity of $100 \mathrm{rad} / \mathrm{s}$ is reached, a steady state periodic solution is observed when looking into the vertical displacement of the top plate of the bearing, as shown in figure 2 (a). Consequently, when looking into the force generated by the exciter see figure 2 (b), it is visible that the force oscillates around the preload value, with peakto-peak amplitude of around $8 \mathrm{kN}$.

The energy balance (see figure 2 (c)) of the model is very good. The artificial strain energy is 0.07 percent of the internal energy of the system, indicating no hourglassing of the model and element mesh size is adequate while the total energy of the system is constant ( $3 \mathrm{~J})$. The observed internal energy in figure 2 (c) is the sum of recoverable elastic strain energy in the assembly through out the simulation and the observed artificial energy includes all energy stored in the hourglass (excessive deformation) resistance. Large values of artificial strain energy will indicate that mesh refinement or other changes to the mesh are necessary in the model. Figure 2 (c), indicates an accurate explicit dynamic analysis where no numerical instabilities occurs in the assembly and thus clearly proves capabilities of the FE model to accurately simulate the characteristics of the modelled exciter.

Table 1. Groovy bearing parameters

\begin{tabular}{ll}
\hline Parameters & Values \\
\hline Number of rows & 1 \\
Number of rolling elements & 16 \\
Contact angle & 90 degrees \\
Pitch diameter & $115.00 \mathrm{~mm}$ \\
Bore diameter & $90.00 \mathrm{~mm}$ \\
Ball diameter & $17.00 \mathrm{~mm}$ \\
Bearing width & $8.00 \mathrm{~mm}$ \\
Race diameter & $140.00 \mathrm{~mm}$ \\
Race curvature factor & 0.52 \\
Preload & $6400 \mathrm{~N}$ \\
Rotation & $100 \mathrm{rad} / \mathrm{s}$ \\
Density & $7850 \mathrm{~kg} / \mathrm{m}^{3}$ \\
Elastic modulus & $2.1 \mathrm{e}+11 \mathrm{Mpa}$ \\
\hline
\end{tabular}


(a)

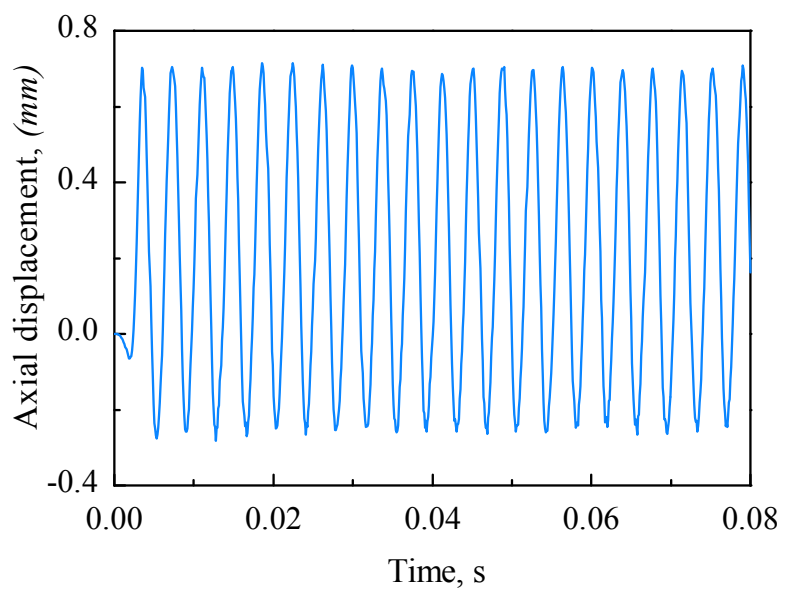

(c)

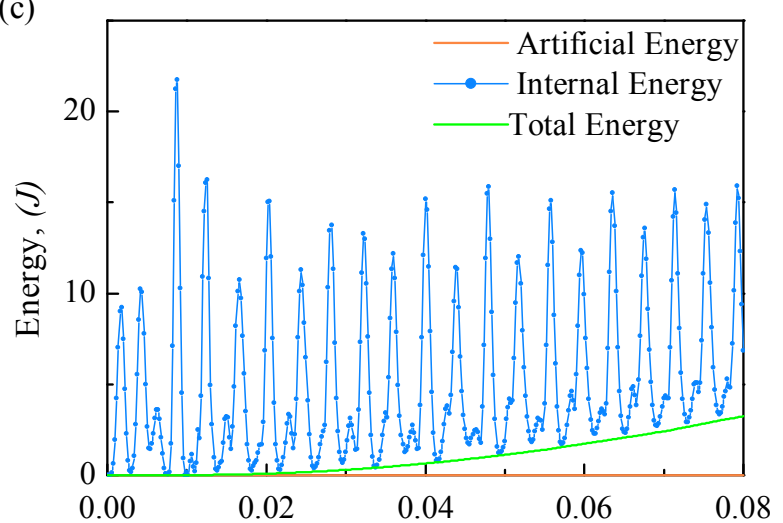

(e)

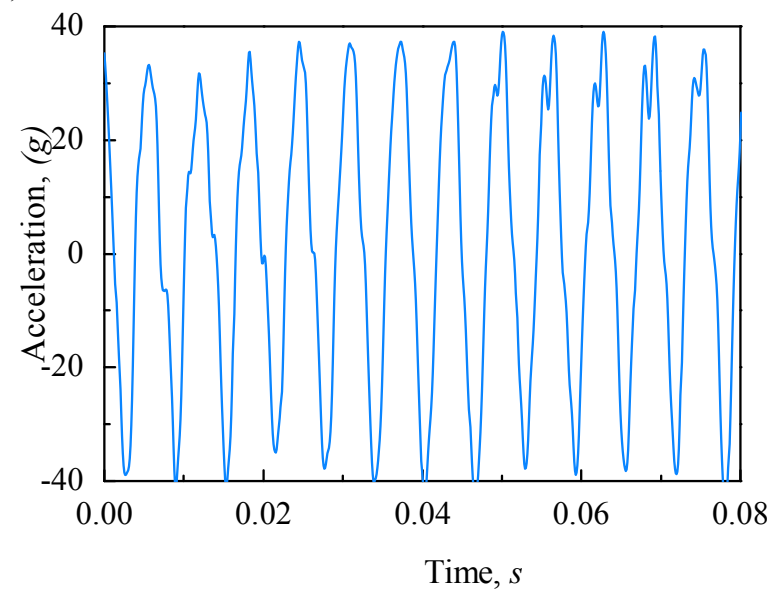

(b)

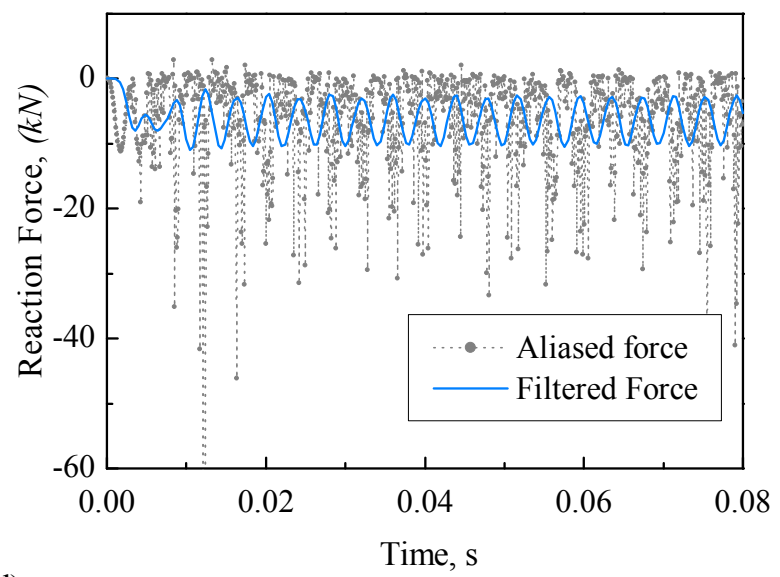

(d)

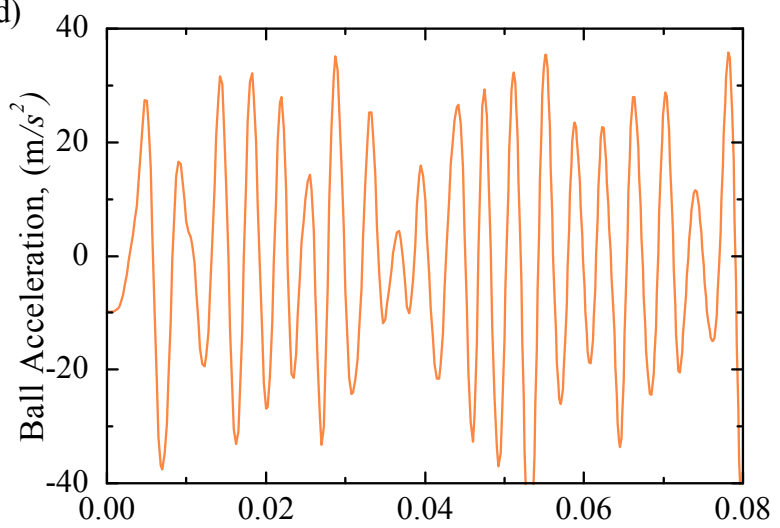

(f)

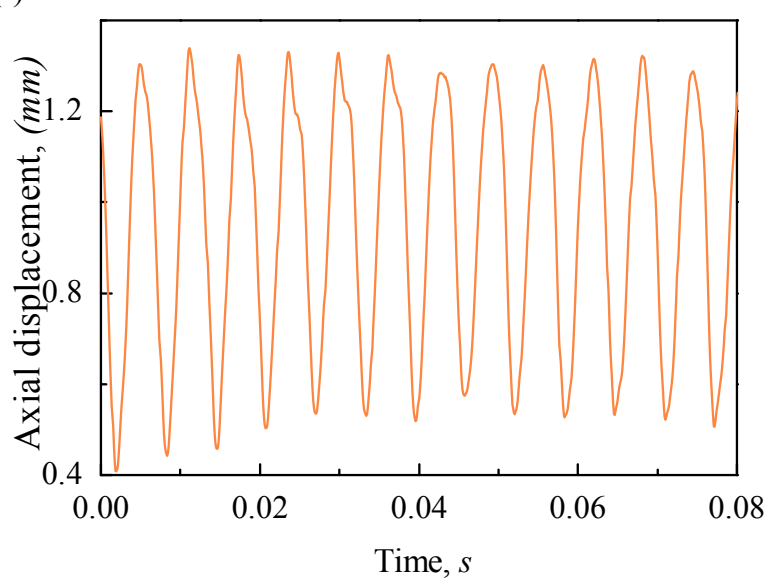

Figure 2. Time history of response of the exciter numerical model and RED experimental result. (a) Axial oscillation of the exciter; (b) Reaction force of exciter; (c) Energy history of the model; (d) Tangential acceleration of the rolling element of FE model; (e) Generated acceleration of the assembly from experiment; (f) Generated displacement of the assembly from experiment. 


\subsection{Experimental verification}

In order to validate the numerical model, the results (axial displacement, reaction torque, force and acceleration) of the finite element analysis have been compared against available RED experimental results in [1]. A typical example of this, is the comparison of the acceleration and displacement from the FE model with experimental result.

Looking at figure $2(\mathrm{e})$, it is observed that the measured experimental acceleration of the system oscillates at 40 $\mathrm{m} / \mathrm{s}^{2}$, as the balls interact with the cage pockets and the race of the bearing, this is in good agreement with the acceleration obtained from the numerical model presented in figure $2(\mathrm{~d})$.

Furthermore, the measured experimental displacement (see figure 2 (f)), shows a $1 \mathrm{~mm}$ peak-peak axial oscillation of the assembly, an exact match to the numerical result presented in figure 2 (a). The results of the experimental validation clearly shows that the numerical model accurately reflects the mechanics of the exciter analysed.

\section{Summary and Future Work}

Modelling of the dynamics groovy ball bearing is undertaken in this study, and the results of the numerical analysis and validation are presented. A parametric study of the developed models has been performed considering different modulus, preload value, amplitude of wave on the groovy race, contact load and other system parameters. The performance characteristics, capabilities and limitation of the developed model under varying excitation loads and initial conditions have been considered.

The next stage of this study, is development of a low dimensional analytical model for optimum design of groovy ball bearing, to improve the performance of the bearing over its service life and geometry optimization of the design.
In conclusion, a finite element contact mechanics bearing model is established based on a contact algorithm suited to high-precision elastic bodies and well-established for rolling element contact mechanics. Appropriate boundary conditions to simulate the mechanics of the bearing being modelled are adopted and load dependent rolling element contact for race-ball and cage-ball interactions is solved using appropriate algorithm in Abaqus.

The numerical model fully captures all the important groovy bearing geometry and demonstrates the capability of the assembly to produce $1 \mathrm{~mm}$ peak-to-peak axial oscillation under applied excitation frequency. The accuracy of the developed model is validated via FEM energy balance equation and experimental result.

\section{Acknowledgements}

This work is performed using Maxwell High Performance Computing (HPC) cluster of the University of Aberdeen IT Service (www.abdn.ac.uk/stanet/research/researchcomputing), provided by Dell Inc. and supported by Alces Software.

\section{References}

[1] M. Wiercigroch, M. Kapitaniak, and V. Vaziri. RED: Revolutionary Drilling Technology for Hard Rock Formations, SPE-184665-MS, Hague, (2017).

[2] M. Wiercigroch, M. Kapitaniak, V. Vaziri and N. Yari. RED Rotary Actuator. Patent No. WO 2016142537 A3 (2016).

[3] O. C. Zienkiewicz. The finite Element Method. McGraw-Hill, (1977).

[4] B. S. Simulia. Abaqus Analysis Users Guide Vol I-IV. Providence, RI, USA, (2012). 